\title{
1 From Luttinger to Fermi liquids in organic conductors
}

\author{
T. Giamarchi \\ DPMC-MaNEP, University of Geneva, 24 Quai Ernest Ansermet, 1211 Geneva, \\ Switzerland \\ Thierry.Giamarchi@physics.unige.ch
}

This chapter reviews the effects of interactions in quasi-one dimensional systems, such as the Bechgaard and Fabre salts, and in particular the Luttinger liquid physics. It discusses in details how transport measurements both d.c. and a.c. allow to probe such a physics. It also examine the dimensional crossover and deconfinement transition occurring between the one dimensional case and the higher dimensional one resulting from the hopping of electrons between chains in the quasi-one dimensional structure.

\subsection{Introduction}

Organic conductors, such as TMTTF and TMTSF compounds offer unique challenges. Indeed from the theoretical point of view most of our understanding of interacting electronic problems is based on Landau's Fermi liquid (FL) theory [1-3]. However it is well known that the effects of interactions can be greatly enhanced by reduced dimensionality. In one dimension, interactions destroy the Fermi liquid and lead to a quite different state known as a Luttinger liquid (LL) [4]. For commensurate systems such as the organic conductors, interactions can also lead to a Mott insulating (MI) state, another state showing clearly the effects of strong correlations.

The organic conductors are thus natural candidates to search for the existence and properties of such states. However because of their very three dimensional nature, they provide not a single one dimensional electron gas, but a very large number of such one dimensional systems coupled together. This allows for a unique new physics to emerge where the system is able to crossover from a one dimensional behavior to a more conventional three dimensional one [5]. This richness is also a drawback, since it is now an important issue to know whether MI or LL physics can be realized at all in these systems. Although for the members of the TMTTF family it was soon undisputable that they are indeed Mott insulators and that interaction effects were important [6], the situation was far from being clear for the TMTSF compounds, that were behaving as good metals with many characteristics of a nice Fermi liquid. Despite important efforts no convincing experimental case could be made for LL behavior and the nature of the normal phase and of the crossover scale between a one dimensional and higher dimensional behavior 
of these compounds remained hotly debated. Understanding the nature of the normal phase and the effect of interactions in these compounds, in addition of being important in its own right, was of course potentially crucial in connection with the low temperature ordered phase and in particular of the superconducting one.

Theoretical progress in computing the transport properties $[7,8]$ and corresponding experimental measurements of the optical conductivity $[9,10]$ allowed for a solution of this dilemma and proved the LL properties of the above mentioned organic conductors. In addition, this led to a definite reexamination $[5,8]$ of what was commonly believed as the main reason [11] for the Mott insulating nature of the parent compounds, namely the dimerization of the organic chain. It also allowed to clearly determine the crossover scale between the one and higher dimensional behavior in these systems, leading to a very consistent understanding of the physics of these materials as well as the one of quarter filled compounds $[6,12,13]$.

I will thus focus in this chapter on the issue of the transport in quasi-one dimensional organic conductors and how it can be used to probe for the MI and LL physics, and more generally on the question of dimensional crossover and deconfinement between the low dimensional Luttinger liquid or Mott insulator and a more conventional high dimensional metal. I mostly concentrate here to the specific applications to the organics here and refer the reader to the review of C. Bourbonnais and D. Jerome [6] for a general introduction, specific experimental data and references on the quasi-one dimensional organics and to previous literature for more details on the derivations $[4,7,8]$, further theoretical issues $[5,8]$ and references.

The plan of this chapter is as follows. In Section 1.2 I review the basic questions and concepts for a system of coupled one dimensional chains. In Section 1.3 I discuss the transport properties of isolated chains and how one can use them to probe for MI and LL physics, as well as various characteristics of the interactions in these systems. In Section 1.4 I discuss effects specifically due to the coupling between the chains, such as the deconfinement transition and some transverse transport properties. Finally conclusions and perspectives are presented in Section 1.5 .

\subsection{General ideas}

Let me first summarize the main ideas and challenges in connection with the quasi-one dimensional nature of the organics, and the observation of LL behavior.

The chains are characterized by intra $\left(t_{\|}\right)$and interchain $\left(t_{\perp}\right)$ single particle hopping. The main effect of the interchain single particle hopping is to induce a dimensional crossover between a one dimensional situation and

a higher dimensional one. In the absence of interactions such dimensional crossover is easy to understand. In Fourier space the kinetic energy becomes 


$$
\epsilon\left(k_{\|}, k_{\perp}\right)=-2 t_{\|} \cos \left(k_{\|} a\right)-2 t_{\perp} \cos \left(k_{\perp} b\right)
$$

where $b$ denotes a perpendicular direction. If the perpendicular hopping $t_{\perp}$ is much smaller than the parallel one $t_{\|}$, which is the relevant case for the quasi-one dimensional organics, then (1.1) leads to the open Fermi surface of Fig. 1.1. If one is at an energy scale (for example the temperature $T$
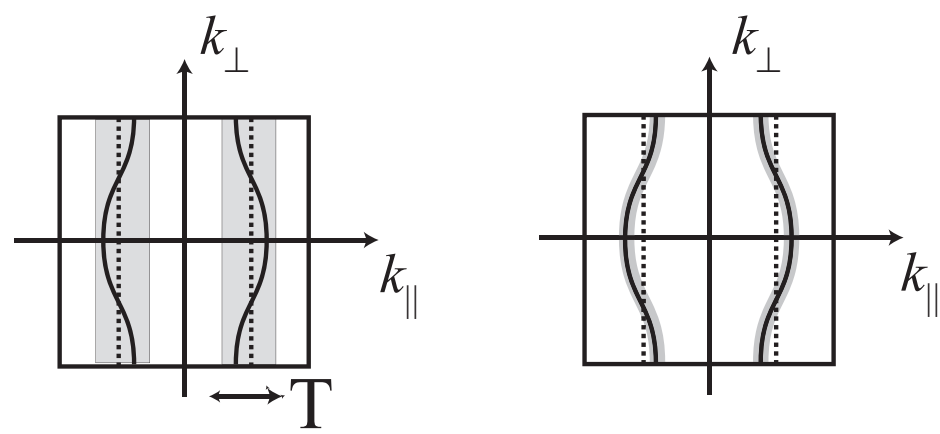

Fig. 1.1. Dimensional crossover for noninteracting electrons. $k_{\|}$is the momentum along the chains and $k_{\perp}$ the one perpendicular to the chains. (left) If the temperature $T$ (or any other external energy scale, represented by the gray area) is larger than the warping of the Fermi surface due to interchain hopping the system cannot feel the warping. It is thus behaving as a one-dimensional system. (right) At a lower temperature/energy the system feels the two (or three) dimensional nature of the dispersion and thus behaves as a full two (or three) dimensional system. There is thus a dimensional crossover as the temperature/energy is lowered.

or the frequency $\omega$ ) larger than the warping of the Fermi surface then the warping is washed out, which means that no coherent hopping can take place between the chains. In that case the system is indistinguishable from one with a flat Fermi surface and can thus be considered as a one-dimensional system. On the other hand if the temperature or energy is much smaller than the warping of the Fermi surface all correlation functions are sensitive to the presence of the warping, and the system is two- or three-dimensional. Since I considered free electrons in the above example, this crossover occurs at an energy scale of the order of the interchain hopping, as is summarized in Fig. 1.1. In presence of interactions and commensurability, the problem is of course much more complicated and interactions affect drastically this behavior compared to the non interacting case. Indeed, for the organics $t_{\perp}$ is much smaller than the intrachain characteristic energy scales such as the kinetic energy or the interactions. In that case the chains can experience the full effect of the interaction in a one dimensional regime before the processes due to interchain hopping can spoil this pure one-dimensional physics. The 
dimensional crossover scale must thus be computed from the one dimensional interacting theory. This is summarized in Fig. 1.2,

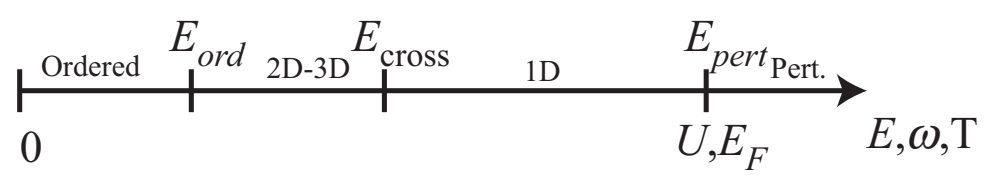

Fig. 1.2. Separation of energy scales if the interchain hopping $t_{\perp}$ is much smaller than the intrachain one $t_{\|}$. For energies larger than the intrachain hopping (or equivalently the Fermi energy $E_{F}$ ) and interactions (denoted generically $U$ ), simple perturbation theory is valid. Below this scale the system is in an interacting one dimensional regime. The interchain hopping couples the chains at an energy $E_{\text {cross }}$ and destroys the one-dimensional physics. For non-interacting particles $E_{\text {cross }} \sim t_{\perp}$ but this scale is renormalized by interactions into $t_{\perp}^{\nu}$ in a LL. For commensurate systems (Mott insulators) the Mott gap can suppress the single particle hopping and drive $E_{\text {cross }}$ to zero. In all cases the system can have a transition to an ordered state at an energy $E_{\text {ord }}$. If the dimensional crossover takes place before (as is the case shown in the above figure), this transition should be described from the twoor three- dimensional interacting theory.

For the case of a commensurate system, the situation is even more complicated and a phase diagram as a function of the temperature (or another energy scale probing the system) and interchain hopping $t_{\perp}$ is shown in Fig. 1.3 If the chains are uncoupled, $t_{\perp}=0$, one recovers the behavior of one dimensional interacting electrons. For an incommensurate system this gives the well known Luttinger liquid behavior, that I will discuss in more details in Section 1.3. The organics are commensurate systems, and thus at low temperature one can expect to have a Mott insulator behavior. An important question, that I will discuss further in Section 1.3 concerns the origin of this Mott insulating behavior. Indeed the material has a quarter filled band (of holes) [6], but a small dimerization of the order of $\Delta_{d} \sim 100 \mathrm{~K}$ makes the band effectively half filled. One has thus to determine which commensurability is important for the Mott behavior. Regardless of this point, one must get an insulating behavior for an isolated chain at low temperature, characterized by a Mott gap in the charge excitation spectrum $\Delta_{\rho}$. For temperatures $T \ll \Delta_{\rho}$ one thus sees the Mott insulating behavior. This is the region represented as (MI) in the phase diagram of Fig. 1.3. For temperatures larger than the Mott gap $T \gg \Delta_{\rho}$ the Mott behavior is not observable and one has a crossover to the Luttinger liquid behavior in the isolated chain. This is denoted as (LL) in the phase diagram of Fig. 1.3.

The interchain coupling brings the system from the one dimensional behavior to a higher dimensional one. However, if each chain develops a gap, it means that the single-particle Green's function decays exponentially. The single-particle hopping is now an irrelevant variable. The formation of a gap 


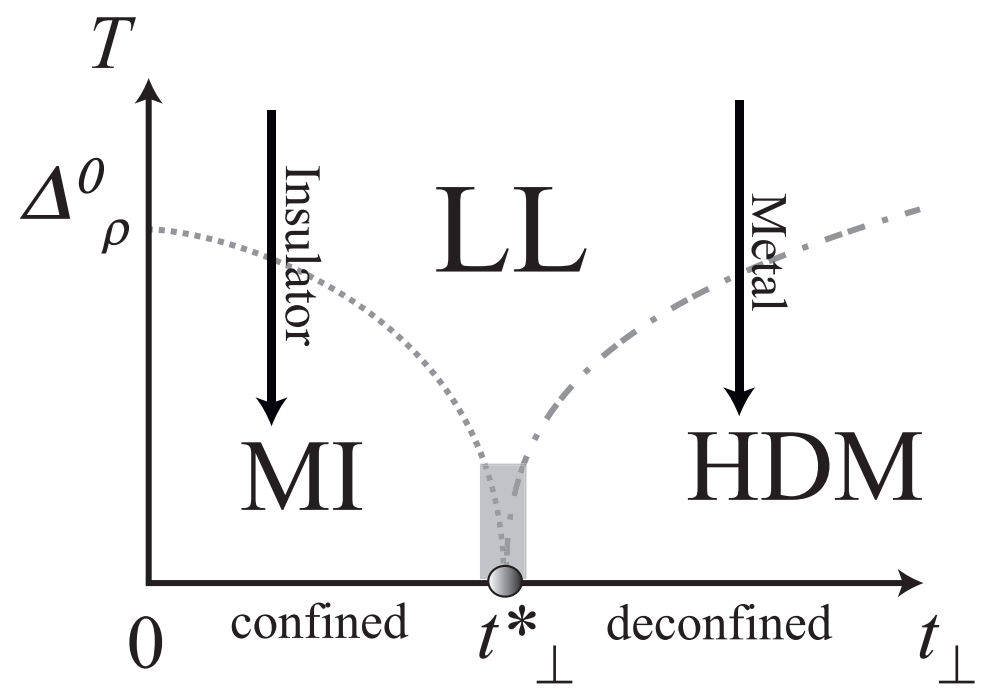

Fig. 1.3. Schematic phase diagram expected for coupled one dimensional commensurate chains as a function of the temperature $T$ (or an energy $E$ ) and the interchain hopping $t_{\perp}$. In the absence of $t_{\perp}$ the system is a one dimensional Mott insulator with a Mott gap $\Delta_{\rho}^{0}$. If $t_{\perp}$ is weak the ground state is still a Mott insulator (MI). For temperature larger than the Mott gap $\Delta\left(t_{\perp}\right)$ (dotted line) one observes a crossover to a Luttinger liquid (LL) regime. Beyond a critical value $t_{\perp}^{*}$ the system has a deconfinement transition towards a high dimensional metal (HDM). Additional complications can occur near this point as schematically represented by the gray box (see text). Above a certain crossover scale $T^{*}$ (dash-dotted line) the LL behavior is recovered since no coherent hopping can take place between chains. These two crossovers can be observed in different materials or upon application of pressure by lowering the temperature as indicated by the two arrows. The two confined and deconfined regions correspond respectively to apparent insulating and metallic behavior when the temperature is lowered. After $[5,14]$.

is thus in direct competition with the interchain hopping. For small interchain hopping the system thus remains an insulator but with a smaller gap $\Delta_{\rho}\left(t_{\perp}\right)$ than for an isolated chain. As far as single particle hopping is concerned the system is thus essentially one dimensional. This is the regime corresponding to the insulating part of Fig. 1.3 As shown in Fig. 1.2 the system can still in this regime undergo a transition to an ordered state if particle-hole (density-density) or particle-particle (Josephson coupling) interactions are present between the chains. Such interactions are in any case generated to second order in the single particle hopping.

The competition between the Mott physics and the interchain hopping means that by increasing the interchain hopping to a critical value one can break the one-dimensional Mott gap. Thus at $T=0$ a quantum phase tran- 
sition occurs for $t_{\perp}=t_{\perp}^{*}$ above which the insulating Mott state is destroyed and the system becomes a high dimensional metal. This transition is known as a deconfinement transition where both the nature of the state as well as the effective dimensionality of the system change. The properties and consequences of this transition will be discussed in Section 1.4. Beyond this critical value of $t_{\perp}$ the low temperature properties are the ones of the high dimensional metal (HDM). What is the nature of such a high dimensional metal (and in particular whether it is a Fermi liquid or not) and how it is affected by the fact that it stemmed from coupled one dimensional chains is of course an important question. As the temperature is increased above a temperature $T^{*}\left(t_{\perp}\right)$ one can expect a crossover towards a one dimensional LL behavior again. Indeed at high temperature coherent hopping between the chains cannot take place. This dimensional crossover energy scale is drastically affected by both the interactions present in the chains and the commensurability and is thus quite different from the one for noninteracting electrons.

This generic phase diagram is directly relevant for the Q1D organics [6] due to their commensurate nature. In particular they are very good realization of quasi-one dimensional systems with hopping integrals of the order of $t_{a} \simeq 3000 \mathrm{~K}, t_{b} \simeq 300 \mathrm{~K}, t_{c} \simeq 20 \mathrm{~K}$, leading to relatively well separated energy scales in which one is indeed dominated by the intrachain hopping. The band is quarter filled, with a small dimerization along the chains giving some half filled character to the system as well. The various parameters $\left(t_{\|}, t_{\perp}\right.$ and the dimerization) can be tuned either by changing the chemistry of the compound or by applying external pressure, so the phase diagram of Fig. 1.3 can be roughly seen as a temperature (or energy) - pressure phase diagram. I will come back to the role of pressure in Section 1.3 Experimentally, at ambient pressure, the (TMTTF) ${ }_{2} \mathrm{PF}_{6}$ compound displays an insulating behavior (MI). A transition to a metallic phase is found, with increasing pressure and the properties of the TMTTF compounds evolve toward those of the compounds of the TMTSF family, which are good conductors. This evolution is clear from the a-axis resistivity measurements (see e.g. Fig. 1.5 of [6]). Such an insulating behavior is well consistent with what one would expect for a one-dimensional Mott insulator. The minimum of the resistivity (followed by an activated law as temperature is lowered) defines the onset of the MI regime on Fig. 1.3 It is thus clear that the interactions play a crucial role in the TMTTF family even at relatively high energies. For the TMTSF, the question is more subtle in view of the metallic behavior at ambient pressure and it was even suggested that such compounds could be described by a FL behavior with weak interactions [15]. On the contrary, interpretations of deviations of $1 / T_{1}$ in NMR [16] or magnetoresistance [17] as due to a one-dimensional behavior would suggest that one dimensional effects would persist to temperatures as low as $20 K$, a much too low scale compared to the naive one given by the bare interchain hopping $t_{b} \sim 300 \mathrm{~K}$. The TMTTF and TMTSF 
family thus prompts for very fundamental questions in connection with one dimensional physics:

1. Are interactions also important for the metallic members of the family or can they be simply regarded as a Fermi liquid with an anisotropic Fermi surface?

2. If indeed one can identify Luttinger liquid behavior, what are the Luttinger parameters?

3. If the system is a Mott insulator is this mostly due to the dimerization of the band or is the quarter filling commensurability sufficient?

4. What are the deconfinement scale $t_{\perp}^{*}$ and beyond that point the crossover scale $T^{*}$ below which the system is not one dimensional any more? What is the nature of the high dimensional metallic phase?

I will now show how transport measurement, and specially optical conductivity, have proven to be a key tool in addressing and to a large extent answering these important questions.

\subsection{Mott insulators and 1D transport}

Let me first examine the properties of the system in a regime where the coherent hopping between the chains can be neglected. This is the regime corresponding to the LL and MI parts of Fig. 1.3 In that regime the properties are essentially the ones of isolated chains, and in particular the transport properties along the chains can be computed from a pure one dimensional limit.

\subsubsection{Theory of transport}

I here recall only the salient points on transport in connection with the quasione dimensional organics and refer the readers to $[4,5,7,8]$ for more details on the transport in one dimension and references.

If the filling is not commensurate, all excitations of a one dimensional system are sound waves of density and spin density. A convenient basis to describe such a system is provided by the so-called bosonization technique [4]. The energy of these excitations is given by a standard elastic-like Hamiltonian. The Hamiltonian of the system is the sum of a part containing only charge excitations and one containing only spin excitations.

$$
H=H_{\rho}+H_{\sigma}
$$

where $H_{\nu}(\nu=\rho, \sigma)$ is of the form

$$
H=\frac{1}{2 \pi} \int d x\left[u_{\nu} K_{\nu}\left(\pi \Pi_{\nu}(x)\right)^{2}+\frac{u_{\nu}}{K_{\nu}}\left(\nabla \phi_{\nu}(x)\right)^{2}\right]
$$


$\phi_{\nu}$ and $\Pi_{\nu}$ are conjugate variables $\left[\phi_{\nu}(x), \Pi_{\nu}\left(x^{\prime}\right)\right]=i \hbar \delta\left(x-x^{\prime}\right)$. The fields $\phi_{\nu}$ are related to the long wavelength distortions of the charge $\rho(x)$ and spin $\sigma(x)$ electron density by $\rho(x), \sigma(x)=-\sqrt{2} \nabla \phi_{\rho, \sigma}(x) / \pi \cdot u_{\rho, \sigma}$ are the velocities of these collective excitations. In the absence of interactions $u_{\rho}=u_{\sigma}=v_{F}$. Interactions of course renormalize the velocities of charge and spin excitations, as in higher dimensions. $K_{\rho, \sigma}$ are dimensionless parameters depending on the interactions. For systems with spin rotation symmetry $K_{\sigma}=1$ (for repulsive interactions) while the spin excitations are gapped for attractive interactions. $K_{\rho}=1$ in the absence of interactions and quite generally $K_{\rho}<1$ for repulsive ones. The three parameters $u_{\rho}, u_{\sigma}$ and $K_{\rho}$ completely characterize the low energy properties of a one dimensional system. They can be computed for a given microscopic model as a function of the interactions [18-23], but as was shown by Haldane [24-26], the form (1.2) is the generic low energy form. This means that (1.2) and the parameters $u_{\rho}, u_{\sigma}, K_{\rho}$ play a role similar to the one of the Landau Fermi liquid Hamiltonian (and Landau parameters) in higher dimensions. To have again in one dimension a concept equivalent to the Fermi liquid, i.e. a generic description of the low energy physics (for energies lower than $E_{\text {pert }}$ of Fig. (1.2) of the interacting problem, is of course extremely useful. This removes part of the caricatural aspects of any modelization of a true experimental system and allows to easily deal with extensions such as the commensurability with the lattice.

The form (1.2) immediately shows that an excitation that is looking like a free electron (i.e. that carries both charge and spin) cannot exist. This is a very important difference between a Luttinger and a Fermi liquid since in the latter, in addition to collective modes of charge and spin, individual excitations (quasiparticles) carrying both charge and spin and looking essentially like a free electron do exist [1-3]. In addition, the correlation functions in a LL display non universal power laws with exponents dependant on the interactions via the Luttinger parameter $K_{\rho}$. For example the single particle correlation function decays with distance or time with an exponent $\zeta=\frac{1}{4}\left[K_{\rho}+K_{\rho}^{-1}\right]+\frac{1}{2}$. The fact that it decays faster than $1 / r$ which is the case for free electrons or a Fermi liquid shows directly that single particle excitations do not exist in one dimension. Similarly spin-spin or density-density correlations have a $2 k_{F}$ oscillating part decaying with an exponent $K_{\rho}+1$. Probing such power laws is thus a direct proof of the LL behavior.

For commensurate systems one has to modify the Hamiltonian (1.2) to take the commensurability with the lattice into account. Such commensurability is at the root of the Mott transition. Although one can of course work out the Mott transition from microscopic models such as the Hubbard model [27], the Luttinger liquid theory provides an excellent framework to take into account the effects of a lattice and describe the Mott transition. It is particularly well adapted for the case of the organics since, as we will see, the Mott gap is smaller than $E_{\text {pert }}$ and thus the LL theory is indeed a suitable starting point at these energies. To incorporate the Mott transition in 
the Luttinger liquid description one must take into account that in presence of a lattice the wavevector is in fact defined modulo a vector of the reciprocal lattice (that is, in one dimension a multiple of $2 \pi / a$ with $a$ the lattice spacing). Thus, in addition to the interaction processes that truly conserve momentum $k_{1}+k_{2}=k_{3}+k_{4}$ one can now have umklapp processes [28] such that $k_{1}+k_{2}-k_{3}-k_{4}=Q$ where $Q$ is a vector of the reciprocal lattice. Since umklapps do not conserve momentum they are the only ones that can lead to a finite resistivity, and are responsible for the $T^{2}$ law in a Fermi liquid [29]. The umklapp process is also responsible for the Mott transition in one dimension. It order for such process to be efficient at the Fermi level it is necessary to have $4 k_{F}=2 \pi / a$ namely $k_{F}=\pi / 2$ or one electron per site (half filling). This corresponds to the case where two electrons are scattered from one side of the Fermi surface $\left(-k_{F}\right)$ to the other side $\left(+k_{F}\right)$. This is indeed the most standard case for having a Mott insulator. But in fact, umklapps are not restricted to one particle per site $[30,31]$, but occur for any commensurate fillings. Indeed, if $2 p k_{F}=2 \pi q / a$ (where $p$ and $q$ are integers) then one can show that an additional term must be added to (1.2). For even commensurabilities $(p=2 n)$, that corresponds to case of the quasi-one dimensional organics) this term is $[7,8,30]$

$$
H_{\frac{1}{2 n}}=g_{\frac{1}{2 n}} \int d x \cos \left(n \sqrt{8} \phi_{\rho}(x)\right)
$$

where $n$ is the order of the commensurability ( $n=1$ for half-filling - one particle per site; $n=2$ for quarter-filling - one particle every two sites and so on). The coupling constant $g_{1 / 2 n}$ is the umklapp process corresponding to the commensurability $n$.

If the bosonization representation can give the universal form of the Hamiltonian and the umklapp term, the amplitude of the umklapp coefficients $g_{\frac{1}{2 n}}$ depends on the precise microscopic interaction. At half filling, for a Hubbard model, $g_{\frac{1}{2}}$ is of the order of the interaction $U$. Higher commensurability umklapps can be estimated perturbatively. For a quarter-filled band such that $8 k_{F}=2 \pi / a$ (this corresponds to $n=2$ in the above notations), to produce an umklapp one needs to transfer four particles from one side of the Fermi surface to the other to get the proper $8 k_{F}$ momentum transfer. This can be done in higher-order perturbation terms by doing three scatterings as shown in Fig. 1.4. For weak interactions the amplitude of such a process would thus be of order $U(U / W)^{2}$, where $W$ is the bandwidth. In addition to the above process, there is an additional one for the Bechgaard or Fabre salt family. Indeed in these systems the stack is slightly dimerised [6,32]. This dimerization opens a gap in the middle of the band as indicated in Fig. 1.4. Thus although the system is originally quarter filled the dimerization turns the system into a half-filled band. This means that even if the system is quarter filled, a non zero $g_{1 / 2}$ exists in addition to $g_{1 / 4}$. If $\Delta_{d}$ is the dimerization gap the strength of such umklapp is $g_{1 / 2}^{d}=U\left(\Delta_{d} / W\right)$. Note that contrarily to what happens in a true half filled system the umklapp coefficient is now much 

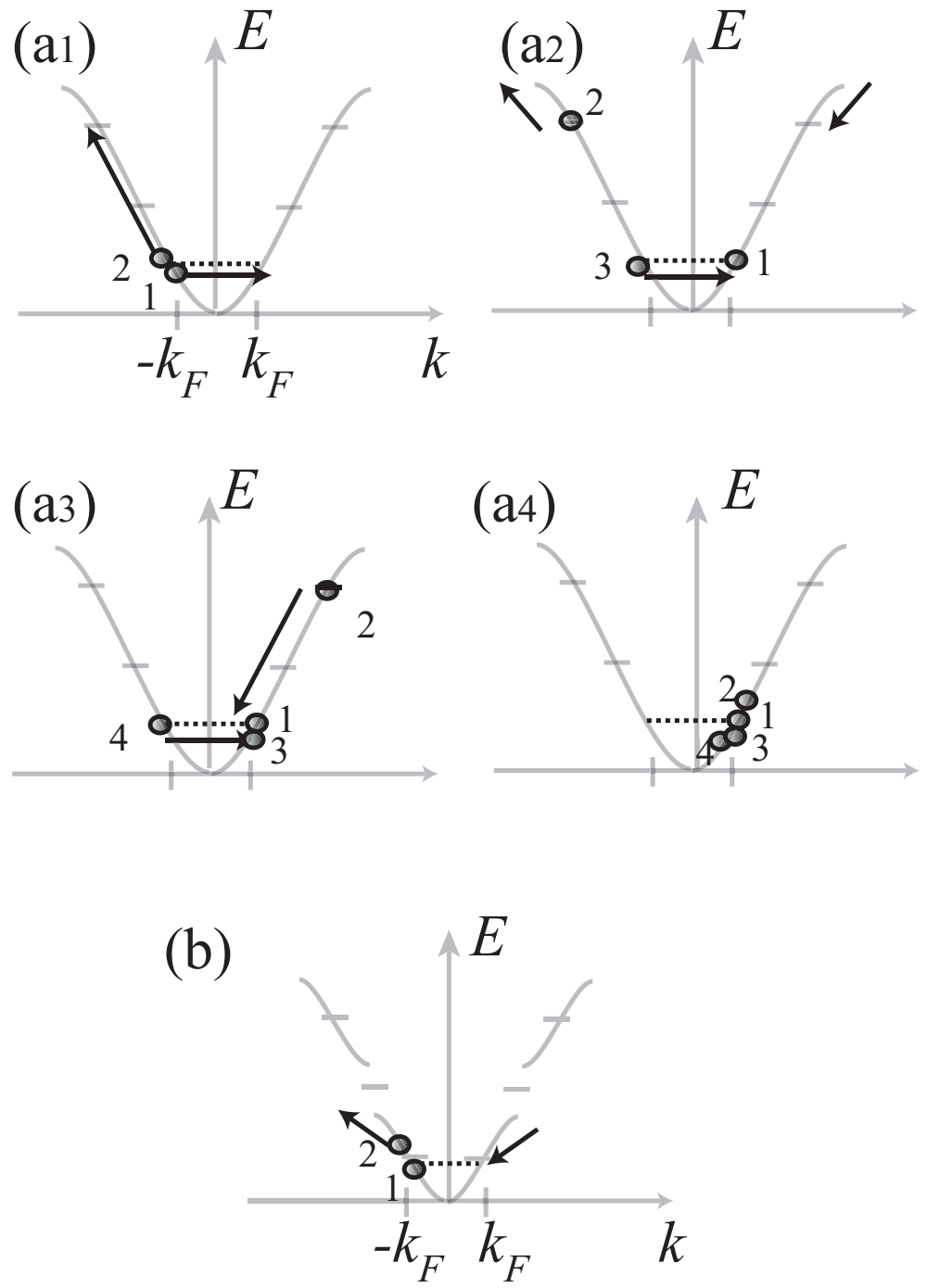

Fig. 1.4. Umklapp processes important for the organic conductors. (a 1-4) A quarter-filled umklapp can be constructed from a third-order perturbation theory in the interaction $U$. It consists in transferring four particles from one side of the Fermi surface to the other. The sequence of scattering due to the interaction is shown in figures (1-4). Three electrons are transferred from $-k_{F}$ to $+k_{F}$ by three successive interaction processes, while the momentum difference is absorbed by a fourth electron until it reaches the opposite side of the Fermi surface. The processus needs two intermediate states of high-energy $W$ of the order of the bandwidth. Thus for small interactions the amplitude for such a process is of order $U(U / W)^{2}$. (b) Dimerization opens a gap in the band. Because of this gap the quarter-filled band becomes effectively half filled. This reduces the zone boundary. The dimerization gap $\Delta_{d}$ thus creates even for a quarter-filled system an half filling umklapp where two particles can be transferred from one side of the Fermi surface to the other. The amplitude of such a process is proportional to the dimerization gap and thus of the order of $U \Delta_{d} / W$. (After [5].) 
smaller than the typical interaction $U$. This allows to get a small Mott gap even if the interactions are large. In presence of dimerization a quarter filled system can thus be a Mott insulator either because of the half-filling umklapp (that exists now because of the dimerization) or because of the quarter-filled one. Which process is dominant depends of course on the strength of the dimerization and of the interactions, and has important consequences on the physics of the system [8].

From (1.4) all the properties of the Mott transition and transport in a one dimensional system can be worked out. The system is a Mott insulator for $K_{\rho}<K_{\rho}^{*}=1 / n^{2}$ where $n$ is the order of the commensurability. The larger the commensurability the smaller $K_{\rho}$ needs to be for the system to become insulating. For a commensurability $n=1$, that is, half-filling the critical value is $K_{\rho}=1$. This means that, contrarily to the higher dimensional case, any repulsive interactions turn the system into an insulator. For a quarterfilled band $(n=2)$ the critical value is $K_{\rho}=1 / 4$. To get the insulator one needs both pretty strong interactions and interactions of a finite range, since the minimum value of $K_{\rho}$ for a local interaction is $K_{\rho}=1 / 2$ [33]. This is physically obvious: in order to stabilize a structure in which there is a particle every two sites one cannot do it with purely local interactions. The range of the interactions in addition of their strength and thus the precise chemistry of the compound controls the range of values of $K_{\rho}$ that one is able to explore.

Of course the Mott transition and the Luttinger physics have drastic consequences on the transport properties and one can expect quite different properties than for Fermi liquid. Thus transport can be used as an efficient probe. As we will see it allows to probe both the single particle behavior (or absence thereof) and the Luttinger liquid collective excitations [7,8,30,34-37]. A schematic plot of the ac conductivity (at $T=0$ ) is shown in Fig. 1.5. In the Mott insulator $\sigma$ is zero until $\omega$ is larger than the optical gap $2 \Delta_{\rho}$. For frequencies larger than the Mott gap, interactions dress the umklapps and give a nonuniversal (i.e. interaction-dependent) power law-like decay. Such a power law can be described by renormalization group calculations coupled to a memory function formalism $[7,8]$. The results of this approach have been subsequently confirmed by form factor calculations [35]. If one ignores the renormalization of $K_{\rho}$ by the umklapp (for the effect of the renormalization of $K_{\rho}$ see [7]) one gets for the a.c. conductivity, for frequencies larger than the Mott gap

$$
\sigma(\omega) \sim \omega^{4 n^{2} K_{\rho}-5}
$$

where $n$ is the order of commensurability. The dc conductivity can be computed by the same methods $[7,8,37]$ and is shown in Fig. 1.6. Here again the dressing of umklapps by the other interactions results in a nonuniversal power law dependence for temperatures larger than the Mott gap $\Delta_{\rho}$. Within the same approximations than for (1.5) one obtains

$$
\rho(T) \sim T^{4 n^{2} K_{\rho}-3}
$$




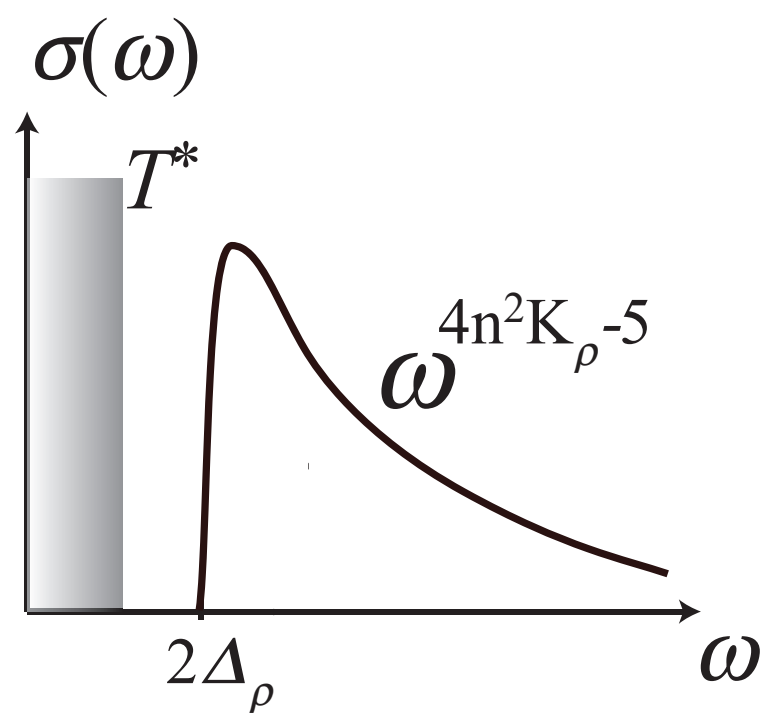

Fig. 1.5. A.c. conductivity along the chains for a commensurability of order $n$. $\Delta_{\rho}$ is the Mott gap. The full line is the conductivity in the Mott insulator (the confined region). Above the optical gap (twice the thermodynamic one $\Delta_{\rho}$ ) the conductivity decays as a power law with an exponent $\mu=4 n^{2} K_{\rho}-5$ characteristic of the Luttinger liquid behavior. A simple band insulator would give $\omega^{-3}$. In the deconfined region most of the features remain, except that below the dimensional crossover scale $T^{*}$ the conductivity is not given by the one dimensional theory any more. The metallic nature corresponds to the appearance of a Drude peak close to zero frequency. This Drude peak must be computed from a two- (or three-) dimensional theory.

\subsubsection{Tranport in the organics}

Independent of any theory, a clear proof of the importance of interactions for both the TMTTF and TMTSF compounds is provided by the optical conductivity $[9,10]$. The optical conductivity shows a decreasing gap (of the order of $2000 \mathrm{~cm}^{-1}$ for the TMTTF $2\left(\mathrm{PF}_{6}\right)$ to $200 \mathrm{~cm}^{-1}$ for TMTSF $2\left(\mathrm{PF}_{6}\right)$. Nearly $(99 \%)$ of the spectral weight is in this high-energy structure. In the metallic compounds there is in addition a very narrow Drude peak (see [6] for additional data). This clearly indicates that these compounds are very far from simple Fermi liquids. Furthermore, the data of optical conductivity can be compared with the theoretical calculations for a one-dimensional Mott insulator (see Fig. 1.5) as shown in Fig. 1.7 The data above the gap fits very well the predicted power law LL behavior (1.5) above the gap and thus shows quite convincingly that these compounds are indeed well described by a LL theory at high energy [10]. This was, to the best of my knowledge, the 

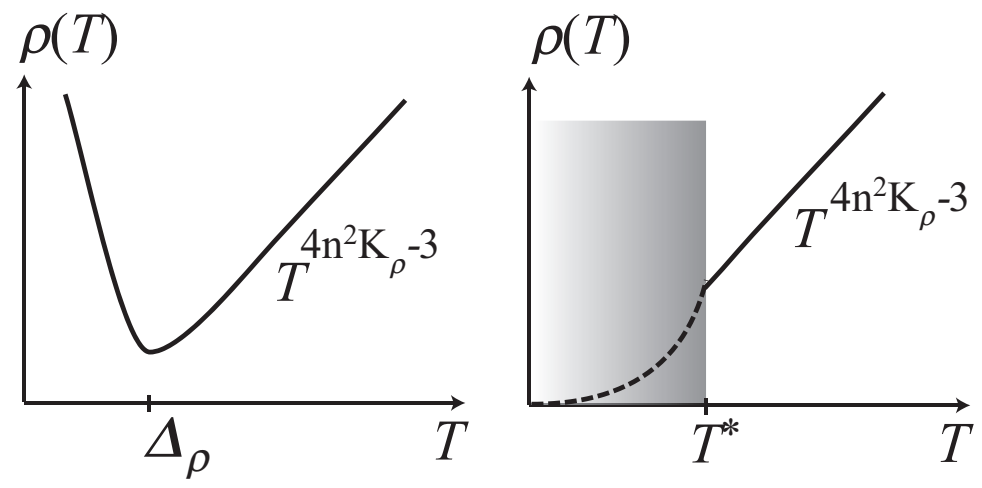

Fig. 1.6. D.c. conductivity along the chains as a function of the temperature $T$ for a commensurability of order $n . \Delta_{\rho}$ is the Mott gap. Left: confined region. Above the Mott gap the dc transport shows an exponent $4 n^{2} K_{\rho}-3$ characteristic of the Luttinger liquid. Below the Mott gap the number of carriers is exponentially small, and any scattering will give an exponentially small conductivity. Right: deconfined region. The Mott gap scale does not exist any more. Above the dimensional crossover scale $T^{*}$ the temperature dependence is essentially identical to the one on the left and shows LL behavior. Below the scale $T^{*}$ the system must be described by a twoor three dimensional theory and one can expect a temperature dependence much more conventional (it would be $T^{2}$ for a simple Fermi liquid).

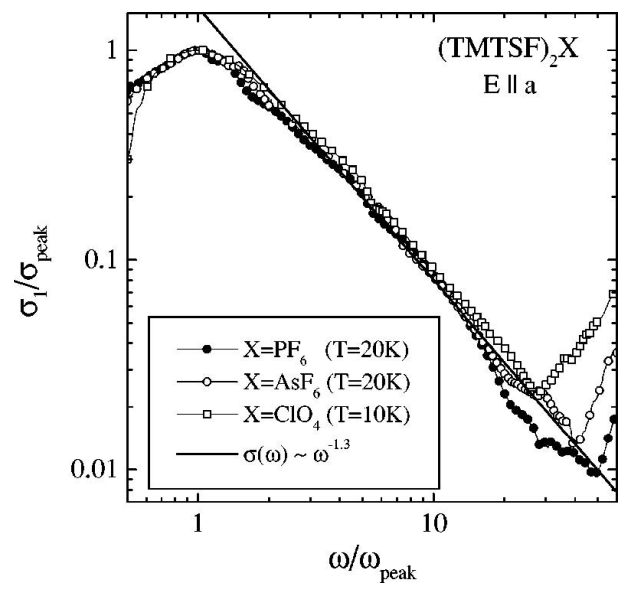

Fig. 1.7. Optical conductivity along the chain axis in the TMTSF family. The conductivity is rescaled by the gap in various samples. A power law behavior is clearly observed. The optical conductivity thus allows to show that even in the metallic (deconfined) regime LL behavior is still present. Because of the wide frequency range accessible on which the power law is seen it also allows to extract the LL parameter $K_{\rho}$ reliably. (From [10] (Copyright 1998 by the American Physical Society).) 
first direct proof of a Luttinger liquid behavior in an electronic system. This measurement also allows to directly extract the Luttinger liquid parameter $K_{\rho}$. A similar comparison can be done on the dc transport and gives also good agreement $[6,32,38]$ with the predicted power law (1.6). Recent d.c. transport data on (TMTSF) ${ }_{2} \mathrm{PF}_{6}$ [39] are also shown in Fig. 1.8, From the data of Fig. [1.8 one sees that $(\mathrm{TMTTF})_{2} \mathrm{PF}_{6}$ shows a quite consistent behavior with the above theoretical description. For temperatures $T$ larger than about $100 \mathrm{~K}$ one recovers a power-law $\rho_{a} \propto T^{0.56}$ quite compatible, using (1.6) with the value of $K_{\rho}=0.22-0.23$ obtained from the optics. Additional experiments both in optics [40-43] and on dc transport [6,32,39] have confirmed the LL nature, and thus the upper part of the phase diagram of Fig. 1.3.

Quite remarkably, optical conductivity is in fact an excellent probe of the LL behavior. Indeed if the control parameter is the temperature several limitations are present. First the compounds are quite compressible, and it is important to make pressure corrections to compare the theoretical prediction (at constant volume) with the experimental observations [6,32,38]. Not taking into account this volume change with temperature was initially responsible for the seemingly $T^{2}$ behavior observed till room temperature. However the pressure correction is hard to perform with great accuracy and more importantly the range of temperature between $T^{*}$ and room temperature, which is in practice about the maximum range available, is very limited (typically $100-300 \mathrm{~K}$ at best in $\mathrm{PF}_{6}$ ) making difficult a quantitative and convincing test of a power law regime. Optics does not suffer from those limitations. Since the parameter varied is the frequency, no such expansion correction is needed. The range of energy that can be explored is also much larger and limited only by the bandwidth of the system. This allows for a fit of the power law on more than a decade. It is important to note that such an analysis of a.c. transport has also been used with success in other types of one dimensional materials [44]. It is thus a method of choice to probe for the physics of these systems.

Indeed quite importantly the $a$-axis optical measurements described above even allow for a quantitative determination [10] of the LL parameter $K_{\rho}$ and a better understanding of the mechanism behind the Mott transition in these materials. A fit of the frequency dependence of the longitudinal conductivity (see Fig. 1.7) can be performed using (1.5). A commensurability of order one $(n=1)$ does not allow for a consistent fit of both the exponent and the Mott gap [10]. Such a commensurability would lead to $K_{\rho} \sim 1$, a nearly non-interacting system and then to a gap much smaller than the one observed experimentally. This indicates that, at least for the TMTSF members of the family, the dominant umklapp comes from the quarter filled nature of the band. Formula (1.5) with $n=2$ thus yields $K_{\rho} \simeq 0.23$, indicating quite strong electron-electron interactions. Moreover this indicates that the finite range nature of the interactions should be taken into account, with interactions extending at least to nearest neighbors. A modelization of the 


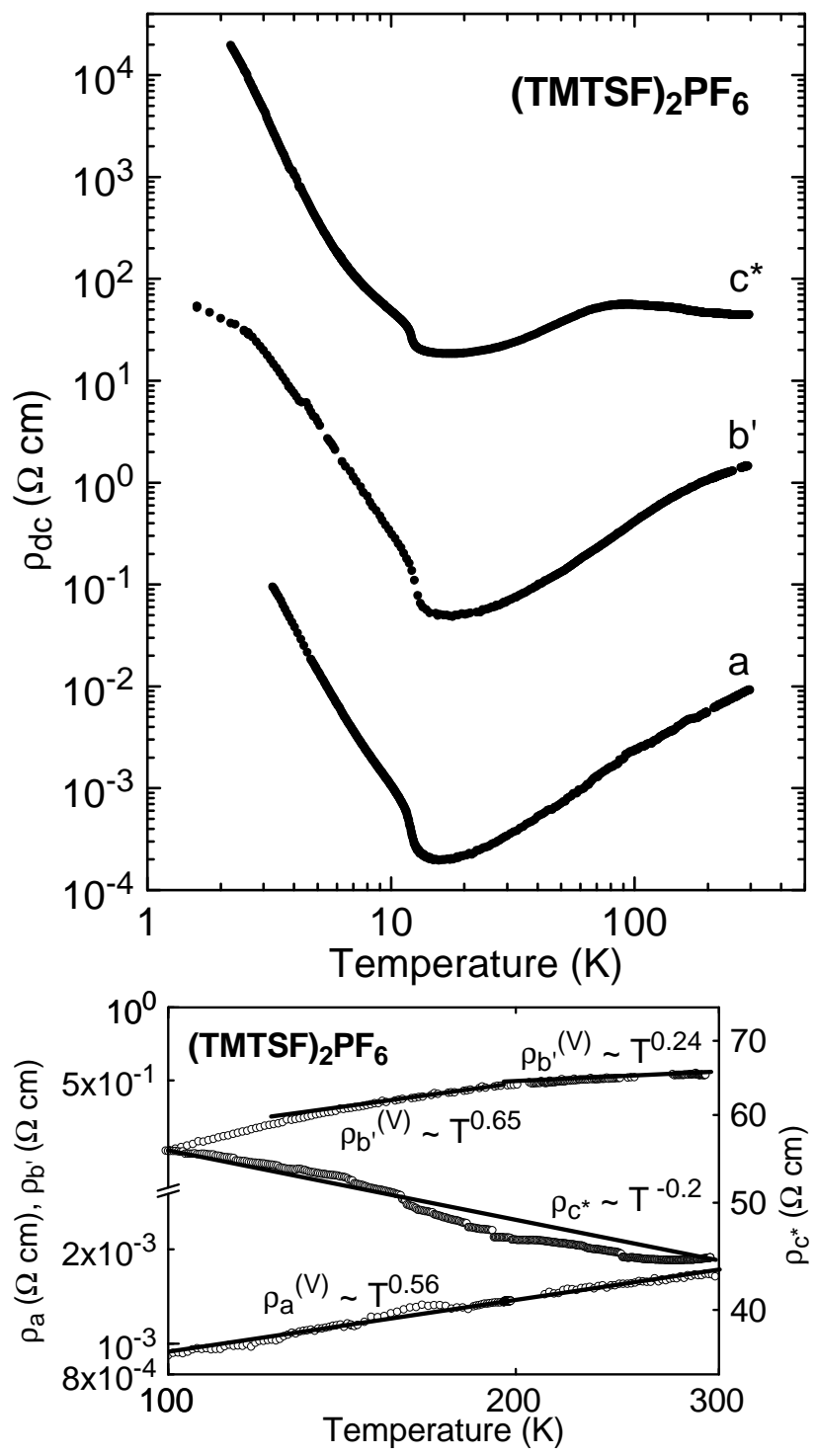

Fig. 1.8. (top) Conductivity of $\mathrm{TMTSF}_{2} \mathrm{PF}_{6}$ at constant pressure. The difference between the $a$ and $c$ axis at high temperature is directly visible and is a signature of the LL behavior. The crossover temperature scale is here slightly below $100 \mathrm{~K}$ as indicated by the change of behavior of $\rho_{c}(T)$. (bottom) The conductivity, corrected to be the constant volume one is shown above the crossover scale $T^{*}$. Both the $\rho_{a}(T)$ and $\rho_{c}(T)$ temperature dependence are well compatible with the value of the LL parameter $K_{\rho} \sim 0.23$ extracted from the optics. The crossover in the $\rho_{b}(T)$ dependence is obviously much broader but the same qualitative tendency than in $\rho_{c}(T)$ can be seen. (From [39] (Copyright 2005 by the American Physical Society).) 
organics thus should not be done with a purely local Hubbard model, with an interaction $U$, but also take into account at least the nearest neighbor interaction $V$. The optical data is thus consistent with an interpretation of the insulating state as a quarter-filled Mott insulator, suggesting that contrarily to what was commonly believed [11] the dimerization plays little role at least in the TMTSF family. In the TMTTF family, dimerization is larger and it is unclear there which process is dominant. Note that because of the anions other transitions can exist such as a ferroelectric transition [45-47]. It is important to note that this also suggests $[5,10]$, that a very important effect of pressure, is not so much to affect the dimerization, but to affect the hoppings and thus reduce the interaction versus kinetic energy ratio. This makes the system effectively less interacting with pressure. This prediction has been recently confirmed on optical measurements under pressure where a consistent decrease of the Luttinger parameter $K_{\rho}$ has been observed with increasing pressure [43]. Given the fact that the value of $K_{\rho}$ is very close to the critical value $K_{\rho}=0.25$ for which the quarter filled umklapp becomes irrelevant, such a variation of $K_{\rho}$ can trigger a rapid variation of the Mott gap upon application of pressure, or when going from the TMTTF members to the TMTSF members. The importance of the quarter filled umklapp in this family of compounds has been also clearly confirmed by properties of parents compounds with a structure similar to the Bechgaard salts but that do not have dimerization $[12,13,48,49]$. These compounds turned out to be Mott insulators [6]. Under pressure they share most of the features of the Bechgaard and Fabre salts, indicating that the same physics is at hand $[13,49,50]$. It would be of course very interesting to further investigate the phase diagram and the transport properties under pressure of these compounds. In addition, since they share the same basis microscopic features, it would be specially interesting to assert whether these quarter filled systems also exhibit superconductivity under pressure as in the Bechgaard salts.

Finally the last question that can be addressed by the transport along the chains, is the one of the value of the crossover scales $\Delta_{\rho}\left(t_{\perp}\right)$ or $T^{*}\left(t_{\perp}\right)$ as shown in Fig. 1.3. $\Delta_{\rho}\left(t_{\perp}\right)$ is easily seen from the upturn of the resistivity along $a$-axis or directly from the optics for the insulating members of the family. In the metallic regime $T^{*}\left(t_{\perp}\right)$ can be estimated by the crossover between a $T^{2}$ behavior at low temperature to the nonuniversal power law (1.6) corresponding to the LL at high temperatures. For example this suggests a crossover scale of about $T^{*} \sim 100 \mathrm{~K}$ for (TMTSF) ${ }_{2} \mathrm{PF}_{6}$ as seen on Fig. 1.8. However a much more precise determination of this scale is provided by a measure of the transverse transport [51] that I now examine.

\subsection{Coupled chains}

Let us now investigate the effects that are direct consequences of the coupling between the chains. There are of course the deconfinement transition at $t_{\perp}^{*}$ 
and the two crossover scales $\Delta\left(t_{\perp}\right)$ and $T^{*}\left(t_{\perp}\right)$. But the very fact that many chains are presents means that transverse transport effects can be probed as well, even in the high energy (LL) of the phase diagram of Fig. 1.3. Such transverse transport is sensitive on how electrons can tunnel from one of the chain to the other. It thus reflects directly how well single particle excitations can exist, and is therefore also a way to probe the LL nature of the system. It is of course also a very sensitive way to address the question of the dimensional crossover since one can expect a drastically different type of transverse transport depending on whether single particle excitations exists in the chain and thus can hop or not.

Let me first discuss the LL region in Fig. 1.3. In that region the hopping is incoherent between the chains. Thus the transverse conductivity can be computed in the high temperature or high frequency regime by an expansion in the perpendicular hopping [52]. One finds a power-law either in frequency of temperature, controlled by the single particle Green's function exponent. At finite temperatures

$$
\sigma_{\perp}(T \gg \omega) \propto T^{2 \alpha-1}
$$

for $k_{B} T \gg E_{\text {cross }}$, while at high-frequency $\left(\hbar \omega \gg E_{\text {cross }}\right)$, one gets

$$
\sigma_{\perp}(\omega \gg T) \propto \omega^{2 \alpha-1}
$$

where $\alpha=\zeta-1=\frac{1}{4}\left(K_{\rho}+K_{\rho}^{-1}\right)-\frac{1}{2}$ is the exponent in the single particle density of states. $E_{\text {cross }}$ is the scale at which this expansion breaks down, either $\Delta_{\rho}\left(t_{\perp}\right)$ or $T^{*}\left(t_{\perp}\right)$ as given by the dotted and dashed lines in Fig. 1.3. Note that in the regime where chains are in the LL state if one takes $K_{\rho} \sim 0.23$ as given by the measurement of the intra chain transport, the transverse conductivity decreases with decreasing temperature or frequency. One has thus a very different behavior of the intra and interchain transport. This is to be contrasted from a normal Fermi liquid regime, where one can expect similar temperature dependencies in both directions. The change of behavior can thus be used to detect the dimensional crossover scale $T^{*}$. This is quite clear on Fig. 1.8

Observed optical conductivity along $c$-axis [42] is compatible with the power law growth of (1.8) and a value of $K_{\rho} \sim 0.23$, as determined by the inchain transport. But clearly much more experiments would be needed since the measurement is extremely difficult and the data not at the same level of accuracy than the intrachain transport. At the price of the pressure correction the dc transport can also be used. For (TMTTF $)_{2} \mathrm{PF}_{6}$, as shown in Fig. 1.8, the temperature dependence of the in-chain conductivity gives, with (1.6), a value of $K_{\rho}=0.22-0.23$ well compatible with the one from the optics. In the same way $\rho_{c} \propto T^{-0.2}$ and (1.7) gives $\alpha=0.69$ again well compatible with $K_{\rho}=0.22$. Note that the fact that the dc transport is at least qualitatively reproduced with the same value of $K_{\rho}$ gives strong credence to a LL physics interpretation of the data. The interpretation of $\rho_{b}$ is more complex since no simple power-law is seen over the entire temperature range. However a 
change in the anisotropy behavior takes place above $T^{*} \sim 100 \mathrm{~K}$ in Fig. 1.8 and the fit to a power-law shows a clear downturn of the exponent. A possible interpretation of the data is thus as being in a crossover regime between the low-temperature Fermi liquid one and the high-temperature Luttinger liquid. Note that it is reasonable to expect a much larger crossover region for the $b^{\prime}$-axis transport, than for the $c^{*}$ axis given the much higher value of the transfer integral in this direction. Despite this general agreement, a quantitative understanding of the transport along $b$ clearly requires further work both for transport [39] and for optics [42].

The temperature dependence below $T \approx 100 \mathrm{~K}$ is the same for the $a$ and $b^{\prime}$ directions implying a similar transport mechanism, and the anisotropy ratio corresponds roughly to the expected band structure value. Below this scale, the dc resistivity follows a power-law $\rho_{a}, \rho_{b} \propto T^{2}$, as expected for a Fermi liquid. Note that although the system is now two dimensional because the hopping in the $b$ direction is now coherent, the temperature is still much larger than the hopping in the $c$ direction $T \gg t_{c}$. Thus one can still use formula (1.7) but putting $\alpha=0$ as would befit a Fermi liquid. This gives $\rho_{c} \propto T$ which is effectively consistent with the experimental data. The experiments in (TMTSF) ${ }_{2} \mathrm{PF}_{6}$ thus directly confirms the above theoretical analysis and the Luttinger liquid behavior. The crossover scale can be experimentally determined to be $T^{*} \sim 100 \mathrm{~K}$ for the case of $\mathrm{PF}_{6}$. For the case of $\mathrm{TMTSF}_{2} \mathrm{ClO}_{4}$ a similar analysis suggests a much higher value $T^{*} \geq 200 K$ [39]. However several problems remain with this compound, in particular concerning the high temperature resistance anisotropy which is much smaller than normally expected. More experimental data is clearly needed in that case. The crossover scale can also be followed under pressure (see Fig. 1.8 of [6]). Another way to determine the crossover scale is provided by the optical $b$ axis conductivity since coherent hopping between the chains manifests itself as the appearance of a Drude peak in the $b$ axis conductivity [42].

Another measurement that can in principle probe the nature of the Luttinger liquid is of course the Hall effect. Indeed in a Fermi liquid the Hall effect is essentially a measure of Fermi surface properties. At low temperatures (in the HDM part of the diagram) the Hall effect can be quite successfully described in this framework [53-55]. At temperatures larger that $T^{*}$ one could expect the Hall effect to reflect again the nature of the interactions in the LL phase. However both the theory and the experiments concerning this quantity are more complicated. Experiments with the magnetic field along the $c$ axis $[56,57]$ observe a weak temperature dependence while field along $a$ leads to an essentially temperature independent Hall effect [58]. On the theoretical side, quite surprisingly it was shown that in the absence of scattering along the chains the Hall effect in a Luttinger liquid does not show any trace of the interactions and is equal to the band value $[59,60] R_{h}^{0}$. Including the scattering along the chains was done recently $[61,62]$ for the case of the half-filled umklapp and magnetic field perpendicular to the chains, leading to a Hall 
effect behaving as

$$
R_{h}=R_{h}^{0}\left[1-A\left(\frac{g_{\frac{1}{2}}}{\pi v_{\mathrm{F}}}\right)^{2}\left(\frac{T}{W}\right)^{3 K_{\rho}-3}\right],
$$

where $A$ is a dimensionless constant and $W$ the bandwidth of the material. This shows that some temperature dependence is to be expected in the Luttinger regime, in qualitative agreement with the observations $[56,57]$.

As one can obtain the crossover temperature $T^{*}$, one can also determine the deconfinement critical value $t_{\perp}^{*}$ from the transport measurements. This can be done for example by monitoring the occurrence of metallic behavior in the $a$-axis (see Fig. 1.8 of [6]). In addition, a measure of the gap extracted from the optical conductivity shows that the change of nature between insulating to metallic behavior occurs when the observed gap is roughly of the order of magnitude of the interchain hopping [40] (see Fig. 1.9). On the theoretical

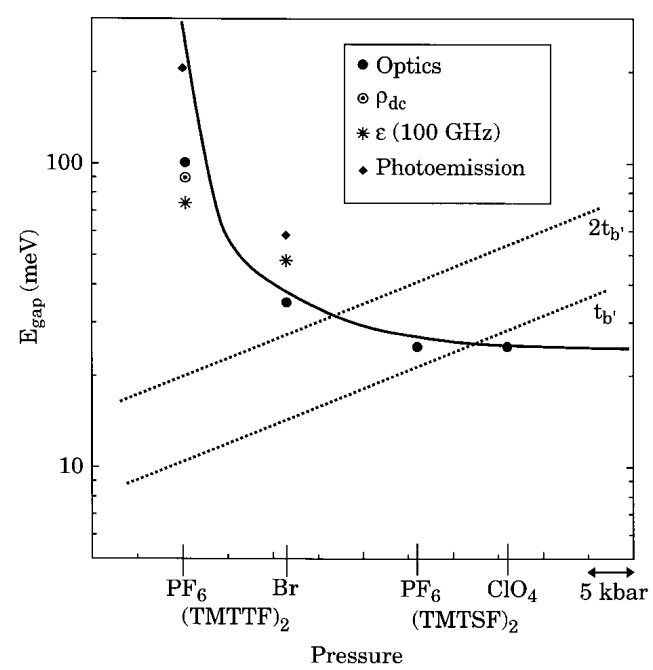

Fig. 1.9. A comparison of the measured gap in the optical conductivity with the interchain hopping. The change of behavior from insulator to metallic occurs when the two quantities are of the same order of magnitude showing that the difference between the various members of the TM families is indeed linked to a deconfinement transition. (From [41] (Copyright 2000 by EDP Sciences).)

side understanding quantitatively the deconfinement transition and even the crossover scale $T^{*}$ for deconfined systems is a major theoretical challenge. In the absence of commensurability, the crossover scale between a LL and the $\mathrm{HDM}$ can be determined by looking at the renormalization of the interchain hopping [63-69]. If one neglects the renormalization of $\alpha$ by the interchain hopping, then one has [64] 


$$
T^{*} \sim W\left(\frac{t_{\perp}}{W}\right)^{\frac{1}{1-\alpha}}
$$

For the non-interacting case $\alpha=0$ and one recovers $E^{*} \sim t_{\perp}$. Since $\alpha$ for an interacting system is always positive, the scale at which the dimensional crossover takes place is always smaller than for free fermions. Interactions thus tend to make the system more one-dimensional. This reduction of the crossover scale comes again from the fact that in a Luttinger liquid single particle excitations are strongly suppressed. For the commensurate case, the scale $T^{*}$ must be computed in presence of the umklapp term, and is thus dependent also on the deconfinement scale. Unfortunately the RG study, although it provides the scale at which the LL is unstable cannot carry easily through the low temperature HDM. What is the nature of this phase is thus still a major challenge. Even if it is a Fermi liquid, since this Fermi liquid stems from the high temperature non-Fermi liquid phase, its features are certainly quite special. In particular the quasiparticle residue $Z$ and lifetime of the quasiparticles could in principle retain the memory of the strong correlations that existed in the one dimensional phase $[5,52]$. In addition since the strength of the hopping depends on the transverse momentum $k_{\perp}$ these quantities could be varying on the Fermi surface and lead to the presence of hot spots [70].

Besides the RG analysis various methods have been tried to tackle the deconfinement transition. This is a difficult problem and much less is known than for the dimensional crossover. Both scaling arguments [8] and study of two chain systems [71-75], showed the importance of the energy scale $T^{*}$ in comparison with the Mott gap $\Delta_{\rho}^{0}$ in the absence of $t_{\perp}$. A rule of thumb to get the position of this deconfinement transition is to compare the two scales $T^{*}$ and $\Delta_{\rho}^{0}$. Thus, roughly if $T^{*}>\Delta_{\rho}^{0}$ one is deconfined, whereas for $T^{*}<\Delta_{\rho}^{0}$ the gap wins and the chains are confined, only allowing for two particles hopping. Of course, this is only a rule of thumb and one should, in principle, solve the full coupled problem to obtain the critical value $t_{\perp}^{*}$ at which deconfinement occurs. No full solution of this problem exists so far. An RG analysis properly incorporating the umklapp terms and the interchain coupling up to the deconfinement transition is quite difficult to realize in a controlled way since both phase correspond to strong coupling fixed points. An RPA treatment [76] of the hopping does produce an insulator-metal transition via the formation of pockets on the Fermi surface. It however neglects any feedback of the hopping on the one dimensional gap itself and thus grossly overestimate the position of the transition. It also cannot give a full deconfinement with an open Fermi surface, since the one-dimensional gap never closes. A quite promising method is a mean field approach (ch-DMFT) treating the chains as an effective bath $[14,52,77-80]$. This method shows clearly the deconfinement transition and gives access to some of the properties of the HDM phase beyond the transition. An analysis has been performed for the half filled case and I refer the reader to $[14,79]$ for more details. The full anal- 
ysis of the quarter filled band, relevant for the quasi-one dimensional organics still remains to be done. A caricature of this case, corresponding roughly to a very large on-site interaction $U$ and a moderate nearest neighbor interaction $V$ can however be performed by considering an half filled band of spinless fermions [80]. In that case a strong depletion of the Mott gap with increasing $t_{\perp}$ has been observed and the deconfinement transition has been analyzed. In such an approach deconfinement occurs first through formations of pockets at a first critical value $t_{c 1}$ but then at a slightly larger value $t_{c 2}$ a full open Fermi surface is recovered. In the HDM phase, effects of the interactions can still be felt, as in particular the presence of hot spots on the Fermi surface [80]. Despite these progress more work both theoretical and experimental is needed to completely understand the deconfinement transition. On the experimental side, it would of course be particularly interesting to have information on single particle excitations. Unfortunately photoemission or STM tunnelling experiments on the organic conductors seems to be difficult due to the ionic nature of the systems and the surface problems it entails. Some results consistent with Mott physics and Luttinger liquids were observed, in particular the Mott gaps $[41,81,82]$. But given the very large energy scales at which for example a depletion of the density of state has been observed, interpretation of these results in terms of LL should be taken with a grain of salt.

The physics below the dimensional crossover scale also remains to be fully understood. However we at least now know reliably from the above mentioned transport experiments the crossover scale to the HDM. This allows to sort out effects that can be attributed to the one-dimensional behavior from those that one has to understand in a more conventional high dimensional system. For the TMTSF members the crossover scale being at least of the order of $\sim 100 \mathrm{~K}$ (for $\mathrm{PF}_{6}$ ) one can expect that a Fermi liquid approach should be a useful starting point much below this temperature. Indeed, Fermi liquid theory has been quite successful in explaining many of the low temperature ordered phase and properties of these compounds (see e.g the chapters on the field induced spin density waves in this book). On the other hand some correlation effects beyond simple Fermi liquids still persists even way below $T^{*}$. This is clear both from the above mentioned theoretical calculations and, from the experimental side, by the existence of anomalies such as the ones in NMR $[16,83]$. Such anomalies, being below $T^{*}$ cannot be attributed to Luttinger liquid behavior. What is their explanation is still an open and a challenging issue.

\subsection{Conclusions and perspectives}

I have presented in this review the main concepts and questions relevant to tackle the normal phase physics of quasi-one dimensional systems. The most important ones for isolated chains are the Luttinger liquid theory and the 
Mott insulating physics which is quite special in one dimension. For quasione dimensional systems an extremely rich physics stems from the coupling between the chains. Its most spectacular expression is the presence of a deconfinement transition between a one dimensional insulator and a high dimensional metal. The quasi-one dimensional organic conductors, provide wonderful systems to investigate these phenomena. But this question is pertinent for many experimental systems and is in particular now investigated in systems such as cold atomic gases as well [84-86].

I have shown here how a good theoretical understanding of the transport properties allows to probe the unconventional physics of these systems. In particular the transport has allowed to prove the Luttinger liquid properties of the quasi-one dimensional organics. It was also instrumental in determining the crossover scale (about $100 \mathrm{~K}$ for $\mathrm{PF}_{6}$ ) between the one-dimensional LL properties and the more conventional high dimensional metallic ones. It also forced to a reexamination of the mechanism underlying the insulating behavior in this compounds showing clearly that the quarter filled nature of the system is enough in itself to lead to an insulating behavior. These findings were confirmed by the existence of non-dimerized compounds with Mott insulating properties. Thanks to these recent progress we have now a consistent description of the relevant properties and energies scales in the quasi-one dimensional organics. This framework now provides a solid reference to focuss on the important still unsolved questions.

Of course the remaining challenges are numerous. The low energy phase properties are still largely not understood, and if some are strongly reminiscent of the ones of a Fermi liquids, some deviate markedly from them, such as the NMR. Even if one has a Fermi liquid it is unlikely to be a plain vanilla one, since it will remember that it stemmed from a low dimensional highly interacting system. This can manifests itself, as is apparent on e.g. the mean field solution, by the variation of the Fermi liquid parameters along the Fermi surface. Much work thus remains to be done to understand this phase, and possibly have a clue on the consequences on the ordered phases, such as the superconducting one. In a similar way the field opened by the new non-dimerized compound must be explored. In particular it is important to determine whether they have indeed the same properties than the TMTTF and TMTSF salts under suitable pressure. No doubts that transport both a.c. and d.c. will prove a useful tool to tackle these systems too. Finally of course, if any for the theorist, since for now no chemist has managed to dope such systems, it would be crucial to complete the phase diagram by adding the doping axis, since most of the questions raised here become even more crucial for doped Mott insulators. 


\subsection{Acknowledgements}

Many people have contributed, directly via enjoyable collaborations, or indirectly via scientific discussions to the work presented here or more generally to my own understanding of the field. The list of the persons I would like to thank would be too long to be given here, but I would like to specially mention: L. Degiorgi, M. Dressel, A. Georges, D. Jérome, H.J. Schulz. Part of this work was supported by the Swiss national fund for research under MaNEP and division II.

\section{References}

1. L. D. Landau, Sov. Phys. JETP 3, 920 (1957).

2. P. Nozieres, Theory of Interacting Fermi Systems (Benjamin, New York, 1961).

3. A. A. Abrikosov, L. P. Gorkov, and I. E. Dzyaloshinski, Methods of Quantum Field Theory in Statistical Physics (Dover, New York, 1963).

4. T. Giamarchi, Quantum Physics in One Dimension (Oxford University Press, Oxford, 2004).

5. T. Giamarchi, Chem. Rev. 104, 5037 (2004).

6. C. Bourbonnais and D. Jerome, 2006, in this book.

7. T. Giamarchi, Phys. Rev. B 44, 2905 (1991).

8. T. Giamarchi, Physica B 230-232, 975 (1997).

9. M. Dressel, A. Schwartz, G. Grüner, and L. Degiorgi, Phys. Rev. Lett. 77, 398 (1996).

10. A. Schwartz et al., Phys. Rev. B 58, 1261 (1998).

11. V. J. Emery, R. Bruinsma, and S. Barisic, Phys. Rev. Lett. 48, 1039 (1982).

12. R. Kato, H. Kobayashi, and A. Kobayashi, J. Am. Chem. Soc. 111, 5224 (1989).

13. K. Heuzé et al., Adv. Mater. 15, 1251 (2003).

14. S. Biermann, A. Georges, A. Lichtenstein, and T. Giamarchi, Phys. Rev. Lett. 87, 276405 (2001).

15. L. P. Gorkov, Physica B 230-232, 970 (1997).

16. C. Bourbonnais et al., J. Phys. (Paris) Lett. 45, L755 (1984).

17. K. Behnia et al., Phys. Rev. Lett. 74, 5272 (1995).

18. A. Luther and I. Peschel, Phys. Rev. B 12, 3908 (1975).

19. H. J. Schulz, Phys. Rev. Lett. 64, 2831 (1990).

20. M. Ogata, M. U. Luchini, S. Sorella, and F. F. Assaad, Phys. Rev. Lett. 66, 2388 (1991).

21. F. Mila and X. Zotos, Europhys. Lett. 24, 133 (1993).

22. N. Kawakami and S. K. Yang, Phys. Lett. A 148, 359 (1990).

23. N. Kawakami and S. K. Yang, Phys. Rev. B 44, 7844 (1991).

24. F. D. M. Haldane, J. Phys. C 12, 4791 (1979).

25. F. D. M. Haldane, J. Phys. C 14, 2585 (1981).

26. F. D. M. Haldane, Phys. Rev. Lett. 45, 1358 (1980).

27. E. H. Lieb and F. Y. Wu, Phys. Rev. Lett. 20, 1445 (1968).

28. I. E. Dzyaloshinskii and A. I. Larkin, Sov. Phys. JETP 34, 422 (1972).

29. J. M. Ziman, Electrons and Phonons (Clarendon, Oxford, 1962).

30. T. Giamarchi and A. J. Millis, Phys. Rev. B 46, 9325 (1992). 
31. H. J. Schulz, in Strongly Correlated Electronic Materials: The Los Alamos Symposium 1993, edited by K. S. Bedell et al. (Addison-Wesley, Reading, MA, 1994), p. 187.

32. D. Jérome, Chem. Rev. 104, 5565 (2004).

33. H. J. Schulz, Phys. Rev. Lett. 64, 2831 (1990).

34. T. Giamarchi, Phys. Rev. B 46, 342 (1992).

35. D. Controzzi, F. H. L. Essler, and A. M. Tsvelik, Phys. Rev. Lett. 86, 680 (2001).

36. E. Jeckelmann, F. Gebhard, and F. H. L. Essler, Phys. Rev. Lett. 85, 3910 (2000).

37. A. Rosch and N. Andrei, Phys. Rev. Lett. 85, 1092 (2000).

38. D. Jérome, in Organic Superconductors: From (TMTSF $)_{2} P F_{6}$ to Fullerenes (Marcel Dekker, New York, 1994), Chap. 10, p. 405.

39. M. Dressel et al., Phys. Rev. B 71, 075104 (2005).

40. V. Vescoli et al., Science 281, 1191 (1998).

41. V. Vescoli et al., Eur. Phys. J. B 13, 503 (2000).

42. W. Henderson et al., Eur. Phys. J. B 11, 365 (1999).

43. D. Pashkin, M. Dressel, and C. A. Kuntscher, 2006, cond-mat/0608242.

44. Y.-S. Lee, K. Segawa, Y. Ando, and D. N. Basov, Phys. Rev. Lett. 94, 137004 (2005).

45. P. Monceau, F. Nad, and S. Brazovskii, Phys. Rev. Lett. 86, 4080 (2001).

46. S. Brazovskii, J. Phys. IV 114, 9 (2004).

47. S. Brazovskii, 2006, in this book.

48. K. Hiraki and K. Kanoda, Phys. Rev. B 54, R17276 (1996).

49. T. Itou et al., Phys. Rev. Lett. 93, 216408 (2004).

50. T. Itou et al., Phys. Rev. B 72, 113109 (2005).

51. J. Moser et al., Eur. Phys. J. B 1, 39 (1998).

52. A. Georges, T. Giamarchi, and N. Sandler, Phys. Rev. B 61, 16393 (2000).

53. V. M. Yakovenko, Synth. Metal 103, 2202 (1999).

54. V. M. Yakovenko and A. T. Zheleznyak, Synth. Metal 120, 1083 (2001).

55. V. M. Yakovenko, 2006, in this book.

56. J. Moser et al., Phys. Rev. Lett. 84, 2674 (2000).

57. B. Korin-Hamzić et al., Phys. Rev. B 67, 014513 (2003).

58. G. Mihaly, I. Kezsmarsky, F. Zamborsky, and L. Forro, Phys. Rev. Lett. 84, 2670 (2000).

59. A. Lopatin, Phys. Rev. B 57, 6342 (1997).

60. A. Lopatin, A. Georges, and T. Giamarchi, Phys. Rev. B 63, 075109 (2001).

61. G. Leon and T. Giamarchi, J. Low Temp. Phys 142, 315 (2006).

62. G. Leon, C. Berthod, and T. Giamarchi, 2006, cond-mat/0608427

63. S. Brazovskii and V. Yakovenko, J. Phys. (Paris) Lett. 46, L111 (1985).

64. C. Bourbonnais and L. G. Caron, Physica 143B, 450 (1986).

65. X. G. Wen, Phys. Rev. B 42, 6623 (1990).

66. C. Bourbonnais and L. G. Caron, Int. J. Mod. Phys. B 5, 1033 (1991).

67. V. M. Yakovenko, JETP Lett. 56, 510 (1992).

68. D. Boies, C. Bourbonnais, and A.-M. S. Tremblay, Phys. Rev. Lett. 74, 968 (1995).

69. H. J. Schulz, in Correlated Fermions and Transport in Mesoscopic Systems, edited by T. Martin, G. Montambaux, and J. Tran Thanh Van (Editions frontières, Gif sur Yvette, France, 1996), p. 81. 
70. A. T. Zheleznyak and V. M. Yakovenko, Synth. Metal 70, 1005 (1995).

71. Y. Suzumura, M. Tsuchiizu, and G. Grüner, Phys. Rev. B 57, R15040 (1998).

72. M. Tsuchiizu, Y. Suzumura, and T. Giamarchi, Prog. Theor. Phys. 101, 763 (1999).

73. M. Tsuchiizu and Y. Suzumura, Phys. Rev. B 59, 12326 (1999).

74. M. Tsuchiizu, P. Donohue, Y. Suzumura, and T. Giamarchi, Eur. Phys. J. B 19, 185 (2001).

75. K. Le Hur, Phys. Rev. B 63, 165110 (2001).

76. F. H. L. Essler and A. M. Tsvelik, Phys. Rev. B 65, 115117 (2002).

77. E. Arrigoni, Phys. Rev. Lett. 83, 128 (1999).

78. E. Arrigoni, Phys. Rev. B 61, 7909 (2000).

79. S. Biermann, A. Georges, T. Giamarchi, and A. Lichtenstein, in Strongly Correlated Fermions and Bosons in Low Dimensional Disordered Systems, edited by I. V. Lerner et al. (Kluwer Academic Publishers, Dordrecht, 2002), p. 81, cond-mat/0201542.

80. C. Berthod, T. Giamarchi, S. Biermann, and A. Georges, 2006, cond-mat/0602304.

81. B. Dardel et al., Europhys. Lett. 24, 687 (1993).

82. F. Zwick et al., Phys. Rev. Lett. 79, 3982 (1997).

83. W. Yu et al., Int. Review of Modern Physics B 16, 3090 (2002).

84. A. F. Ho, M. A. Cazalilla, and T. Giamarchi, Phys. Rev. Lett. 92, 130405 (2004).

85. T. Stöferle et al., Phys. Rev. Lett. 92, 130403 (2004).

86. M. A. Cazalilla, A. F. Ho, and T. Giamarchi, Phys. Rev. Lett. 95, 226402 (2005). 